\title{
Variação sazonal e abundância de Rynchops niger no estuário de Cananéia-Iguape-Ilha Comprida, São Paulo
}

\author{
Edison Barbieri
}

Biota Neotropica $v 7$ (n2) - http://www.biotaneotropica.org.br/v7n2/pt/abstract?article +bn00207022007

\author{
Recebido em 14/11/06 \\ Versão reformulada recebida em 08/01/07 \\ Publicado em 01/05/07
}

Instituto de Pesca, APTA, Secretária da Agricultura e Abastecimento do estado de São Paulo,

CP 61, CEP 11990-000, Cananéia, São Paulo, Brasil

e-mail:edisonbarbieri@yahoo.com.br

\begin{abstract}
Barbieri, E. Season Variation Abundance of Rynchops niger in the estuary of Cananéia-Iguape-Ilha Comprida, São Paulo. Biota Neotrop. May/Aug 2007 vol. 7, no. 2 http://www.biotaneotropica.org.br/v7n2/pt/ abstract?article+bn00207022007. ISSN 1676-0603.

This work aims to make available basic information about the occurrence and abundance of the Black Skimmer in the Cananéia-Iguape-Ilha Comprida estuary of São Paulo. Black Skimmer are found throughout the year at the study area. Censuses were made from January to December 2005, on a weekly basis. A total of 48 censuses were completed in each of five areas in the estuary. The results show that Black skimmer was a common species at the estuary and the number of individuals varied among the sites. The Black skimmer was more frequent in the mud flats of the Baixio do Arrozal (Cananéia), in the summer and autumn, in the summer-winter in Boqueirão Norte and Sul and in the autumn-winter in the Baixio of the Boguaçu. All the estuary, the population presented accentuated fluctuations along the year, with the largest seasonal abundance in summer, when the production of the Anchoviella lepidonstole increase.

Keywords: Brazil, Charadriiformes, estuary, seasonal variation, Rynchops niger, Black Skimmer, abundance.

\section{Resumo}

Barbieri, E. Variação sazonal e abundância de Rynchops niger no estuário de Cananéia-Iguape-Ilha Comprida, São Paulo. Biota Neotrop. May/Aug 2007 vol. 7, no. 2 http://www.biotaneotropica.org.br/v7n2/pt/ abstract?article+bn00207022007. ISSN 1676-0603.

O objetivo deste trabalho foi avaliar a variação sazonal e abundância do Talha-mar Rynchops niger no estuário de Cananéia-Iguape-Ilha Comprida. Durante o período de janeiro a dezembro de 2005. Foram realizados censos semanais, com auxílio de binóculos em cinco áreas do referido estuário. O Talha-mar foi uma ave constante durante todo o ano no estuário de Cananéia-Iguape-Ilha Comprida. Um total de 48 censos foi realizado em cada uma das cinco áreas escolhidas. Os resultados indicaram que o Talha-mar foi uma espécie comum no estuário de Cananéia-Iguape-Ilha Comprida e o número de indivíduos variou em função da área de estudo. O talha-mar foi mais freqüente observado no Baixio do Arrozal (Cananéia), no verão e outono, no verão-inverno no Boqueirão Sul e Norte e no outono-inverno no baixio do Boguaçu. Em todo o estuário, a população apresentou flutuações acentuadas ao longo do ano, com as maior abundância sazonal ocorrendo no verão, quando a produção da manjuba (Anchoviella lepidonstole) aumenta.

Palavras-chave: Brasil, Charadriiformes, estuário, variação sazonal, Rynchops niger, talhamar, abundância.
\end{abstract}




\section{Introdução}

No estuário de Cananéia-Iguape-Ilha Comprida, localizada no Litoral Sul do Estado de São Paulo, é comum a ocorrência de muitas aves marinhas e de praia (Barbieri \& Mendonça 2005). Estas aves utilizam este ambiente para se alimentarem, descansarem e reproduzirem, o que atesta a importância ambiental da região, tanto para aves marinhas e estuarinas como para as de praia (Barbieri \& Pinna 2007).

O Talha-mar (Rynchops niger) ave comumente observada no referido estuário, é uma espécie neotropical ainda pouco estudada no Brasil, embora sua biologia e ecologia sejam bem conhecidas na América do Norte (veja, p. ex., o livro de Joanna Burger sobre a sp e o account do Birds of North América). Encontrada freqüentemente nos grandes rios e lagos do país, alcançando a região costeira durante migrações sazonais, ocorre nos estuários da região norte até o Rio Grande do Sul; bem como na Argentina, Uruguai, Peru, Venezuela e Guiana, e também no litoral da América do Norte (Belton, 1984; Sick 1997; Rosário 1996; Branco \& Fracasso 2005; Mascarello et al. 2005).

Segundo Kranntz (1989) e Sick (1997) esta ave procria na região amazônica, e recentemente Efe et al. (2001) registrou ninhos nos bancos de areia do Rio Ibicuí, no Rio Grande do Sul. Ocorrem, também em grandes bandos nos meses de verão, na Lagoa do Peixe, no estuário da Lagoa dos Patos e em menor abundância na Praia do Cassino (Vooren \& Ilha 1995, Naves 1999) e zona metropolitana de Porto Alegre (Mascarello et al. 2005). A abundância de Rynchops niger para o litoral de Santa Catarina, têm sido registrada nos estuários do Saco da Fazenda (Itajaí) (Branco 2000; Branco \& Fracasso 2005), na Baía Sul de Florianópolis (Rosário 1996) e nas Praias de Laguna e Navegantes (Schiefler \& Soares 1994). No litoral de São Paulo, Olmos e Silva (2001) registraram em Santos-Cubatão, a presença da espécie ao longo de todo o ano, ocorrendo principalmente entre Abril e Junho.

Esta espécie é freqüentemente encontrada no estuário de Cananéia-Iguape-Ilha Comprida, litoral sul do Estado de São Paulo, em todos os meses do ano, utilizando este ambiente durante as migrações para alimentação e descanso. Entretanto, não há trabalhos publicados que registrem a abundância da espécie para a região. $\mathrm{O}$ objetivo deste trabalho foi registrar a ocorrência e a abundância mensal/sasonal do Rynchops niger no estuário de Cananéia-Iguape-Ilha Comprida durante 2005.

\section{Material e Métodos}

\section{Métodos}

Para a escolha dos locais de amostragens foram considerados: a abundância dos bandos de Talha-mar, a localização geográfica e a possibilidade de acesso ao longo do ano, sendo selecionados cinco localidades: Ponta da Praia do Boqueirão Sul $\left(25,05455^{\circ} \mathrm{S}\right.$ e $47,94221^{\circ} \mathrm{W}$;), Ponta da Praia do Boqueirão Norte $\left(24,68409^{\circ} \mathrm{S}\right.$ e $\left.47,4272^{\circ} \mathrm{W}\right)$, Lagoa da Ilha $\left(24,68155^{\circ} \mathrm{S}\right.$ e $\left.47,43909^{\circ} \mathrm{W}\right)$, Baixio do Arrozal $\left(25,05455^{\circ} \mathrm{S}\right.$ e $\left.47,94221^{\circ} \mathrm{W}\right)$ e Baixio do Boguaçu $\left(24,96865^{\circ} \mathrm{S} ; 47,89753^{\circ} \mathrm{W}\right)$, onde os censos foram realizados simultaneamente (Figura 1).

A pesquisa teve duração de 12 meses, sendo realizada entre Janeiro a Dezembro de 2005, com censos semanais, totalizando 48 amostragens em cada uma das áreas escolhidas. Os censos foram feitos durante maré baixa, o melhor período de acesso dos pesquisadores aos locais escolhidos, através de contagem direta, segundo a metodologia descrita por Bibby et al. (1992), onde o observador em um ponto fixo desenvolveu contagem individual da espécie com auxílio de luneta Bausch \& Lomb (20 x 60), binóculos $(10$ x 50) e $(20 \times 90 \times 180)$. - mesma metodologia utilizada em Cubatão por Olmos e Silva e Silva (2001).

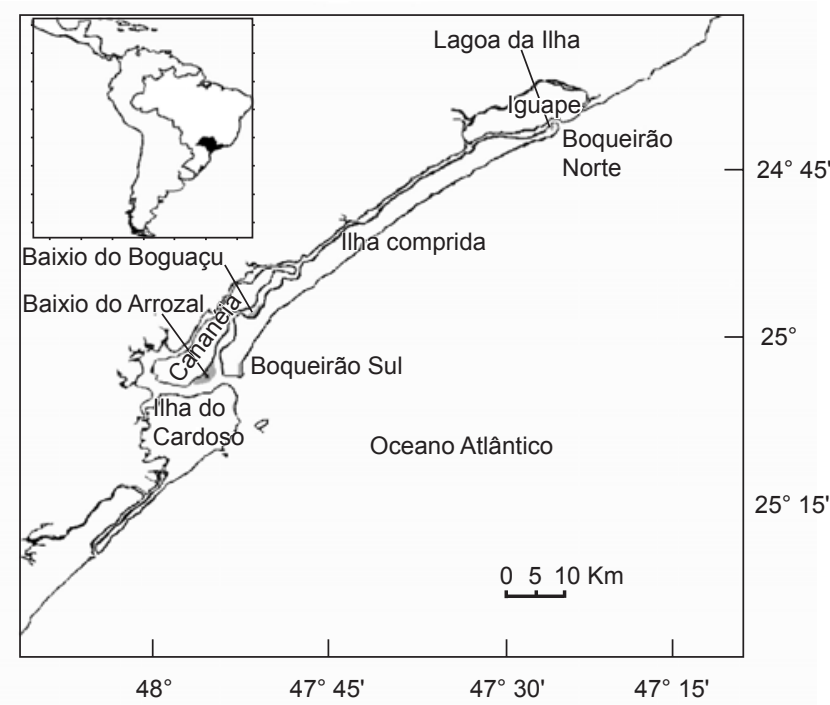

Figura 1. Localização do estuário de Cananéia-Iguape-Ilha Comprida com as áreas de estudo no litoral sul do Estado de São Paulo.

Figure 1. Localization of the Cananéia-Iguape-Iha Comprida estuary with the areas of study in the south coast of the State of São Paulo.

A constância foi calculada com base na fórmula: $\mathrm{C}=\mathrm{p} \mathrm{X} 100 / P$ (Dajoz 1978). Onde $p$ corresponde ao número de visitas que a espécie foi avistada e $P$ refere-se ao número total de visitas. Em função dos valores obtidos, os taxa foram classificados em constates, quando estiveram presentes em mais de 50\% das visitas, acessórios, quando estiveram presentes entre 25 a $50 \%$ das visitas ou, acidentais, quando representaram menos de $25 \%$ das espécies nas visitas. Em todo o estuário a abundância média mensal e sazonal dos talha-mar foram examinadas através de um teste de variância (ANOVA, p > 0,05). Fez-se também a correlação entre os índices pluviométricos mensais, médias da produção de manjuba (Anchoviella lepidonstole) e as médias de $R$. niger. Com a finalidade de se verificar qual dos dois fatores estariam condicionando a presença da ave na região.

\section{Resultados}

Os resultados indicam que o Rynchops niger é uma espécie comum no estuário de Cananéia-Iguape-Ilha Comprida, observada regularmente durante todo o ano, registrando-se a presença dessa espécie em toda a região. Ocorreram em maior número durante o verão e outono. No inverno e na primavera ocorreu uma diminuição no número de indivíduos, em relação às outras estações (Figura 2). Sendo que as maiores ocorrências foram entre os meses de janeiro a março (Figura 3)

No Boqueirão Sul o maior número de indivíduos foi observado em Janeiro e Fevereiro, com 936 e 1196 aves respectivamente, entretanto em Abril e Outubro não houve nenhum registro para a espécie (Tabela 1). Observou-se um declínio da população a partir de Agosto, que permaneceu baixa até Novembro. A população foi acidental em Abril e Outubro, constante de Dezembro a Março e acessória nos demais meses.

De acordo com a Tabela 1 observou-se no Boqueirão Norte, ausência da espécie entre os meses de Fevereiro a Abril e em Novembro e Dezembro respectivamente. Ao contrário da amostragem realizada no ponto anterior, verificou-se que a população foi maior durante o mês de Agosto, com registros de 247 aves. A população foi acidental de Fevereiro a Abril e em Novembro e Dezembro, nos demais meses pode-se classificá-la como acessória. 


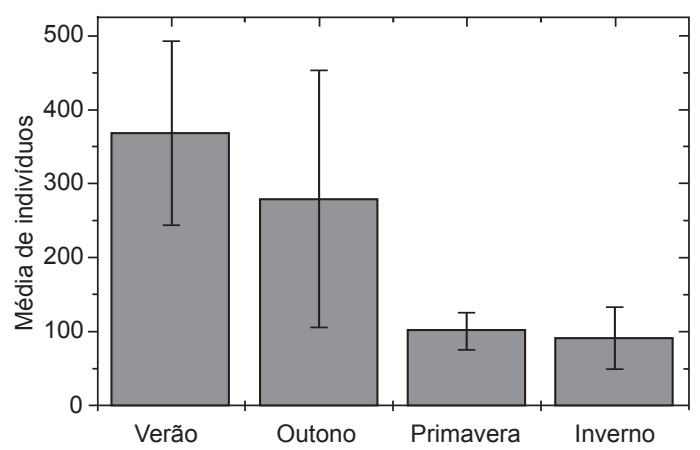

Figura 2. Variação sazonal de indivíduos de Rynchops niger nas cinco áreas combinadas do Estuário de Cananéia-Iguape-Ilha Comprida em 2005.

Figure 2. Seasonal variation of Rynchops niger in the five agreed areas of the Cananéia-Iguape-Iha Comprida estuary in 2005.

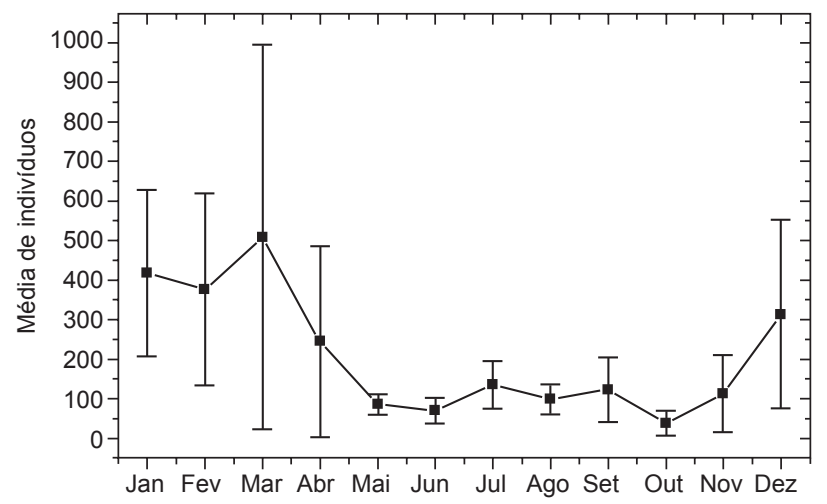

Figura 3. Variação mensal de indivíduos de Rynchops niger nas cincos áreas combinadas do Estuário de Cananéia-Iguape-Ilha Comprida em 2005.

Figure 3. Month variation of Rynchops niger in the five agreed areas of the Cananéia-Iguape-Iha Comprida estuary in 2005.

Na Lagoa observou-se um maior número de Rynchops niger nos meses de Julho, Novembro e Dezembro com 80; 55 e 45 indivíduos respectivamente. A população começou a diminuir a partir de Janeiro e manteve-se baixa durante o verão e o outono, sendo que no mês de Março nenhum indivíduo foi registrado para essa área (Tabela 1). A população foi acessória na maioria dos meses estudados, com exceção do mês de Março quando apresentou-se de forma acidental.

Os censos realizados no Baixio do Arrozal mostraram um aumento gradual da espécie a partir do mês de Janeiro; quando foram avistados 921 indivíduos, 2448 em Março, 1209 em Abril e 1246 em Dezembro (Tabela 1). Houve, no entanto, uma queda dessa população a partir de Maio (148), que se manteve baixa até Agosto, com a menor média registrada (24). Nesta área a população foi constante, durante 8 meses estudados. A população foi constante (acima de 50\%) em quase todos os meses no baixio do Arrozal. Porém, nos meses de Maio e Junho, Agosto e Setembro o número de indivíduos diminuiu, sendo esse número considerado como acessório (25 a 50\%).

Para o Baixio do Boguaçu os menores registros da população ocorreram nos meses de Março e Abril, quando nenhum indivíduo foi registrado (Tabela 1). Esta área foi a que obteve os menores números de indivíduos ao longo de 2005. Embora nos demais meses tenha sido acessória em relação a constância, em todos os outros meses. 
As médias dos cincos pontos combinados mostra uma queda do número de aves a partir de Maio (Figura 2), mantendo-se neste padrão até Novembro. A população de Rynchops niger foi maior durante o verão em relação às demais estações do ano, quando combinados os cinco pontos estudados. Entretanto o mesmo não ocorreu no Boqueirão Norte e na Lagoa da Ilha, onde foi registrado um maior número de indivíduos durante o inverno (Tabela 1).

Foi possível identificar dois locais principais de concentração de Rynchops niger na região estuarina trabalhada. As localidades Arrozal e Boqueirão Sul possuíram meses com médias superiores a 300 e 199 indivíduos presentes respectivamente. Os outros pontos apresentaram médias mensais abaixo de 30 indivíduos da espécie. A região do Boguaçu e Boqueirão Norte, virtualmente foram inexpressivos regionalmente como local de pouso para a espécie, com médias mensais inferiores a 9 indivíduos. Combinando-se os dados de todos os pontos trabalhados em uma única média mensal para a região da Ilha Comprida (Figure 2), nota-se uma sobreposição do desvio padrão mensal, e uma diminuição no número de indivíduos de Maio a Novembro.

Comparando-se as médias sazonais, houve diferença significativa $(\mathrm{p}>0,05)$ entre o verão e o inverno; e entre o verão e primavera. Entretanto, não ocorreu diferenças significativas entre a abundância média mensal de $R$. niger no estuário de Cananéia-Iguape-Ilha Comprida, em 2005.

A correlação entre os índices pluviométricos mensais e as médias de $R$. niger foram forte ( $\mathrm{R}=0,89821, \mathrm{p}=0,00007$, Figura 4). Já a correlação entre as médias da produção de manjuba (Anchoviella lepidonstole $)$ e as médias de $R$. niger não foram forte $(R=0,27243$, $\mathrm{p}=0,39163$, Figura 5 ). Entretanto a variação da produção de manjuba e as médias de Rynchops niger nas cincos áreas combinadas mostra uma tendência das aves acompanharem a abundância do recurso pesqueiro (Figura 6).

Os resultados indicaram que o Talha-mar é uma espécie comum no Estuário de Cananéia-Iguape-Ilha Comprida com ocorrência em todos os meses estudados. Os dados obtidos revelam que o estuário em 2005 foi utilizado durante todo o período das visitas pelo Talha-mar, verificando a presença da espécie nos extremos sul e norte durante todo o ano em grandes bandos, sendo que sua ocorrência, na parte central da Ilha Comprida pode ser considerada rara.

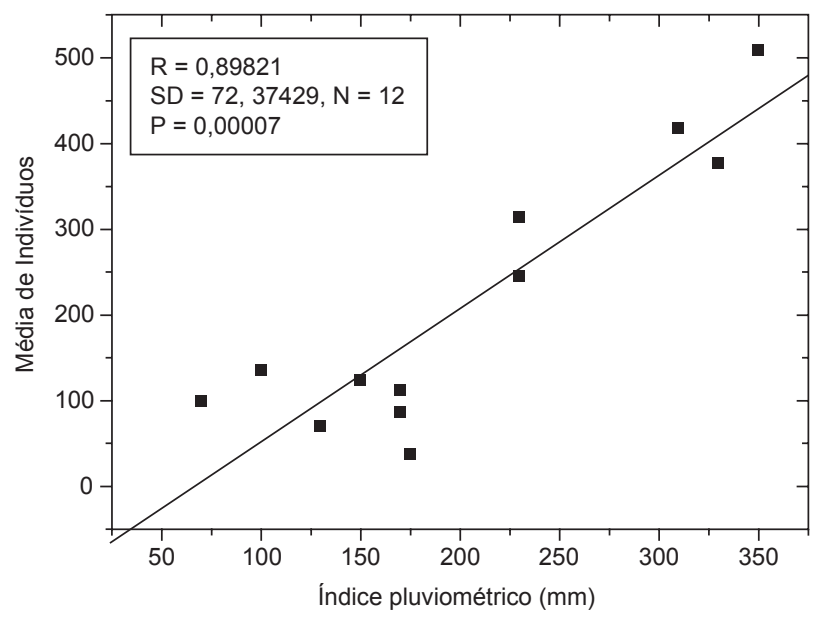

Figura 4. Correlação entre os índices pluviométricos e a média de indivíduos de Rynchops niger nas cincos áreas combinadas do Estuário de CananéiaIguape-Ilha Comprida em 2005.

Figure 4. Correlation between the pluviometrics indices and the average of Rynchops niger in the five agreed areas areas of the Cananéia-Iguape-Iha Comprida estuary in 2005.

\section{Discussão}

As grandes variações apresentadas nos censos mensais para $R$. niger são comuns, pois as aves fazem intensos movimentos de uma área a outra em busca de locais de alimentação e descanso, o que resulta em grandes flutuações na abundância. Este fato já foi também documentado por Barrames \& Pereira (1992), Barbieri \& Mendonça (2005) e Barbieri e Pinna (2007) para Laridae e Scolopacidae.

A diminuição no número de $R$. niger entre Maio e Novembro, registrada no estuário da Cananéia-Iguape-Ilha-Comprida, é diferente da observado por Rosário (2004), que demonstrou uma diminuição da população entre Agosto e Janeiro em Santa Catarina. Já em re-

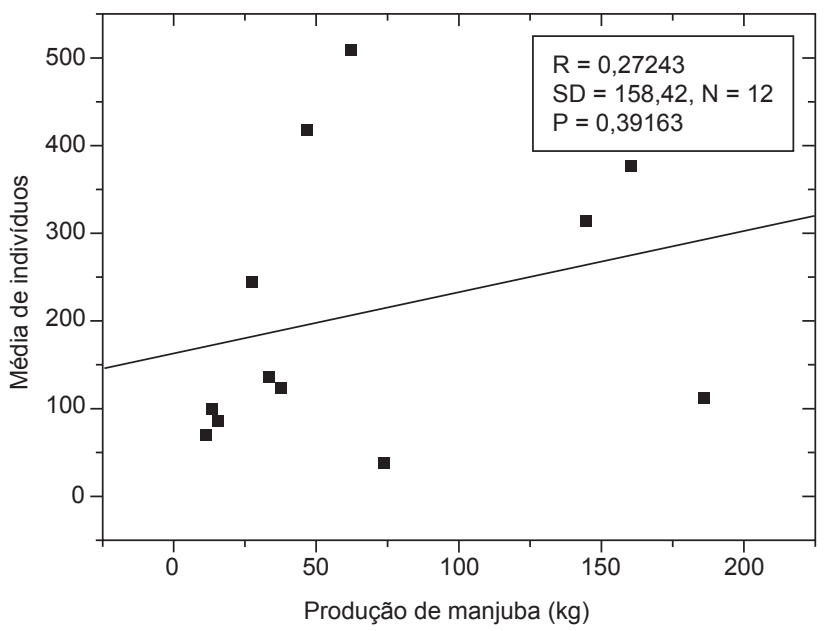

Figura 5. Correlação entre a produção mensal de manjuba (Anchoviella lepidonstole) do município de Iguape e as média de indivíduos de Rynchops niger nas cincos áreas combinadas do Estuário de Cananéia-Iguape-Ilha Comprida em 2005.

Figure 5. Correlation of the monthly production of Anchoviella lepidonstole in Iguape city and the average of Rynchops niger in the five agreed areas of the Cananéia-Iguape-Iha Comprida estuary in 2005.

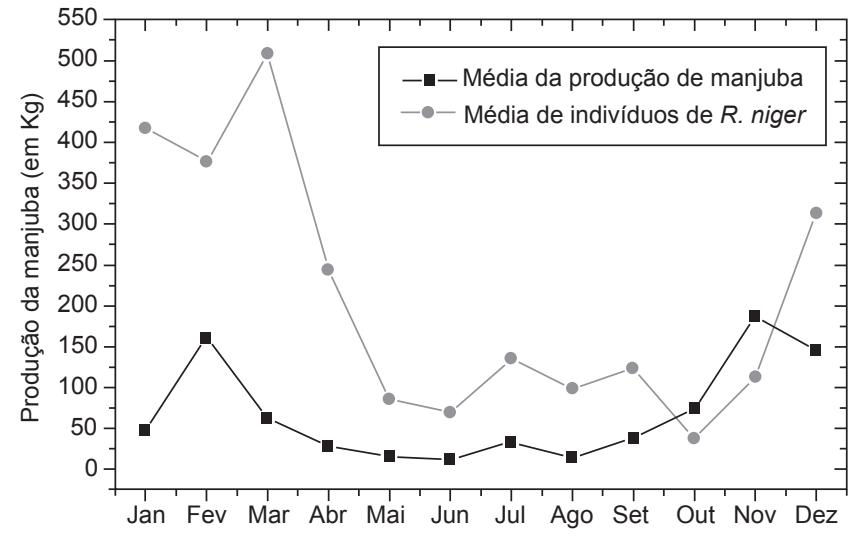

Figura 6. Variação da produção de manjuba (Anchoviella lepidonstole) e das médias de indivíduos de Rynchops niger nas cincos áreas combinadas do Estuário de Cananéia-Iguape-Ilha Comprida em 2005.

Figure 6. Variation of the production of Anchoviella lepidonstole and the averages of Rynchops niger in the five agreed areas of Long Cananéia-Iguape-Ilha Comprida estuary in 2005. 
lação à sazonalidade, o maior número de indivíduos de $R$. niger no presente trabalho, ocorreu durante o verão, diminuindo no inverno e primavera, diferente do registrado por Branco \& Fracasso (2005), para Santa Catarina, quando o pico da população foi maior no Outono e Inverno.

O pico populacional em Santos-Cubatão, onde a espécie também ocorre ao longo de todo o ano, ocorreu entre Abril e Junho, sugerindo que as aves se concentram na área no outono-inverno antes de migrarem para outra área, talvez para nidificar aproveitando as praias fluviais que surgem na bacia do Paraná-Paraguai (Olmos e Silva 2001). A maior abundância no verão e outono no estuário de Cananéa-Iguape e Ilha Comprida, sugere que as aves se originam dos grandes rios interioranos (Paraná-Paraguai), onde nidificam no inverno austral, que é o período seco e com menores vazões. Por outro lado, este é o período de maior pluviosidade no extremo sul do Brasil e as aves dali nidificariam no verão.

Para Vooren \& Chiaradia (1990) os exemplares observados durante os meses de verão na Praia do Cassino (Rio Grande do Sul) pertencem à populações que reproduzem no final do inverno em áreas próximas ou no Rio Grande do Sul. Para Branco \& Fracasso (2005) as observadas em Santa Catarina teriam provavelmente a mesma origem. A ausência de informações sobre a origem das populações que ocorrem ao longo do ano no estuário de Cananéia-Iguape-Ilha Comprida recomenda futuros trabalhos com técnicas de anilhamento para conhecermos a origem das populações que freqüentam o estuário, que foi objeto do presente estudo.

Durante o dia, os bandos de talha-mar foram observados pousados nas coroas arenosas das praias e baixios do estuário, cuidando da plumagem e descansando. Comportamento semelhante foi observado nas populações do litoral catarinense (Rosário 1996; Branco \& Fracasso et al. 2005) e gaúcho (Vooren \& Chiaradia 1990). Na América do Norte a espécie nidifica, na região litorânea em bancos de areia e praias arenosas (Burger 1982; Erwin 1977), enquanto que na América do Sul, ocupa as margens expostas dos grandes rios (Sick 1997; Escalante 1970; Klimaitis \& Moschione 1984).

Na costa do Rio Grande do Sul, Rhynchops niger pousam em bandos monoespecíficos, em lugares fixos (Vooren \& Chiaradia 1990; Naves 1999). O mesmo comportamento foi registrado para o estuário de Cananéia-Iguape-Ilha Comprida, entretanto, $R$. niger foi observado com maior freqüência em bandos heteroespecíficos juntamente com Thalasseus sandivicens.

A espécie não nidifica no litoral paulista, mas parece que a presença da ave no estuário de Cananéia-Iguape-Ilha Comprida nos meses mais chuvosos está relacionado a presença da manjuba. Podendo ser exatamente este o recurso que os talha-mares (e outras aves marinhas) estão explorando na região. Pelos dados, é possível verificar que a ponta sul da Ilha Comprida abriga concentrações de Talha-mar muito mais expressivas do que a ponta norte, além de demonstrar a presença da espécie ao longo de todos os 12 meses do ano. Os sítios fixos mais importantes de pouso de Rhynchops niger para o estuário de Cananéia-Iguape-Ilha Comprida são: Arrozal e Boqueirão Sul. Destes o mais expressivo é o baixio do Arrozal, um baixio de areia onde as aves permanecem durante os doze meses do ano, em repouso sexual. O baixio do arrozal é plano sem vegetação, situa-se no ambiente estuarino, possui margens protegidas nas quais as aves pousam, e é rodeado por águas rasas e calmas com elevada abundância de pequenos peixes estuarinos, que são a base alimentar das aves (Naves 1999). O acesso ao local, só é feito através de embarcações e ainda pouco visitado por pessoas. Existe certamente um limite da tolerância das aves diante da perturbação humana, e quando este limite for ultrapassado no referido baixio, as aves provavelmente desaparecerão da região. Segundo Vooren \& Brusque (1999), dois requerimentos básicos para o sítio de pouso do talha-mar são: proxi- midade à área de pesca, e baixo nível de perturbação pelo homem Para que Rhynchops niger permaneça como integrante da biodiversidade das regiões onde esta ave ocorre, seus poucos sítios de pouso devem ser preservados. A restrição da presença humana nos sítios de pouso é fundamental para se evitar a diminuição da abundância desta ave no estuário de Cananéia-Iguape-Ilha Comprida.

Gaivotas, trinta-réis e o talha-mar alimentam-se nas águas estuarinas e costeiras, e pousam nas praias em bandos para o descanso e os cuidados da plumagem (Sokal \& Rohlf 1969; Escalante 1970; Vooren \& Chiaradia 1990; Quinn 1990; Naves 1999; Barbieri \& Mendonça 2006). A disponibilidade de praias adequadas para o pouso é condição fundamental para a presença destas aves em determinada região (Barbieri e Pinna 2005). Por tanto essas áreas de pouso deveriam ser mapeadas para fornecer subsídios à políticas de conservação, bem como ter visitação e uso restrito.

\section{Agradecimentos}

Agradecemos ao Dr. Fábio Olmos pela revisão e preciosas sugestões, as quais enriqueceram em muito o manuscrito, e também aos técnicos do Instituto de Pesca da Secretaria da Agricultura e Abastecimento do Estado de São Paulo, Núcleo de Pesquisa do Litoral Sul. Antônio Domingues Pires, Antônio Carlos de Almeida, Eduardo Antônio Hoff, Onésio Veríssimo e Sergio Cunha Xavier pela ajuda e coleta de dados em diversas etapas do trabalho.

\section{Referências Bibliográficas}

BARBIERI, E. \& MENDONÇA, J.T. 2005. Distribuition and abundance of Charadridae at Ilha Comprida, São Paulo State, Brazil. J. Coast. Res. 21:1-10.

BARBIERI, E. \& JMENDONÇA, J.T. 2006. Seasonal abundance and distribution of Larids at Ilha Comprida (São Paulo State, Brazil). J. Coast. Res. In Press.

BARBIERI, E. \& PINNA, F.V. 2005. Distribuição da Batuíra-de-coleira (Charadrius collaris) durante o período de 1999 a 2001 na praia da Ilha Comprida. Rev. Bras. Ornitol. 13:25-31.

BARBIERI, E. \& PINNA, F.V. 2007. Distribution of the Royal Tern (Thalasseus maximus) during 2005 in the Cananéia-Iguape- Ilha Comprida estuary. Ornitología Neotropical. 18(1):99-110.

BELTON, W. 1984. Birds of Rio Grande do Sul, Brazil. Part. 1. Rheidae through Furnariidae. Bulletin of American Museum of Natural History, New York, 178:371-631.

BURGER, J. 1982. The role of reproductive success in colony-site selection and abandonment in black skimmers (Rynchops niger). Auk, Fayetteville, 99:109-115.

BRANCO, J.O. 2000. Avifauna associada ao estuário do Saco da Fazenda. Revista Brasileira de Zoologia, Curitiba, 17(2):387-394.

BRANCO, J.O. \& FRACASSO, H.A.A. 2005. Ocorrência e abundância de Rynchops niger Linnaeus, no litoral de Santa Catarina, Brasil. Revista Brasileira de Zoologia. 22(2):430-432.

DAJOZ, R. 1978. Ecologia Geral. 3. ed. Vozes. Petrópolis

EFE, M.A., BUGONI, L., MOHR, L.V., SCHERER, A., SCHERER, S.B. \& BAIRRO, O.P. 2001. First-known Record of breeding for the black skimmer (Rynchops niger) in a mixed colony in Iticuí river, Rio Grande do Sul state, southern Brazil. International Journal Ornithology, Belo Horizonte, 4(2):103-107.

ERWIN, R.M. 1977. Black skimmer breeding ecology and behavior. Auk, Fayetteville, 94:709-717.

ESCALANTE, R. 1970. Aves marinas del Rio de la Plata y aguas vecinas del Oceano Atlántico. Montevideo, Editora Barrero y Ramos, 199p.

KLIMAITIS, J.F. \& MOSCHIONE, F. 1984. Observaciones sobre nidificacion asociada en Charadrius collaris, Sterna superciliaris y Rynchops niger en el Rio Uruguay, entre rios, Argentina. El Hornero, Buenos Aires, 3:197-202. 
KRANNITZ, P.G. 1989. Nesting biology of black skimmers, large-billed terns, and yellow-billed terns in Amazonian Brazil. Journal of field ornithology, Columbus, 60(2):216-223.

MASCARELLO, N.E., MOREIRA, J.S., WURDIG, J.B.R. \& SANDER, M. 2005. Ampliação da zona de distribuição de Talha-mar (Rynchops niger Linnaeus, 1758) no estado do Rio Grande do Sul, Brasil. Biodiversidade Paxmpeana. 3:19-20. Uruguaiana

NAVES, L.C. 1999. Ecologia alimentar do talha-mar Rhynchops nigra (Aves: Rhynchopidae) na desembocadura da Lagoa dos Patos. Rio Grande: Fundação Universidae do Rio Grande, 158p. (Dissertação de Mestrado).

OLMOS, F. \& SILVA E SILVA, R. 2001. The avifauna of a southeastern Brazilian mangrove swamp. International Journal of Ornithology 4(3/4):135-205.

QUINN, J.S. 1990. Sexual size dimorphism and parental care patterns in a monomorphic and a dimorphic larid. Auk, Fayetteville, 107:260-274.
ROSÁRIO, L.A. 1996. As aves em Santa Catarina: Distribuição geográfica e meio ambiente. Florianópolis, FATMA, 329p.

SICK, H. 1997. Ornitologia Brasileira. Rio de Janeiro, Editora Nova Fronteira, 912p.

SCHIEFLER, A.F. \& SOARES, M. 1994. Estudo comparativo da avifauna das praias de Navegantes e Laguna, Santa Catarina. Biotemas, Florianópolis, $7(1 / 2): 31-45$.

SOKAL, R.R. \& ROHLF, J.F. 1969. Biometry, the principles and practices of statistics in biological research. San Francisco, W.H. Freeman, 776p.

VOOREN, C. \& CHIARADIA, A. 1990. Seasonal abundance and behaviour of costal birds on Cassino Beach, Brazil. Ornitologia Neotropical, Québec, 1:9-24.

VOOREN, C. \& ILHA, H.H. 1995. Guia das aves comuns da costa do Rio Grande do Sul. Imago Maris, Rio Grande, 2(1):1-23.

VOOREN, C.M., \& BRUSQUE, L.F. 1999. As aves do ambiente costeiro do Brasil: Biodiversidade e conservação. www.bdt.org.br/workshop/costa/ plat_continental (acesso em 12/11/2006). 